\title{
criginal Paper
}

\section{SIRT1 and Connexin40 Mediate the} Normal Shear Stress-Induced Inhibition of the Proliferation of Endothelial Cells Co-Cultured with Vascular Smooth Muscle Cells

\author{
Qing-Ping Yao ${ }^{a}$ Ying-Xin $\mathrm{Qj}^{\mathrm{a}}$ Ping Zhang Bin-Bin Cheng Zhi-Qiang Yan Zong-Lai Jiang \\ Institute of Mechanobiology \& Medical Engineering, School of Life Sciences \& Biotechnology, \\ Shanghai Jiao Tong University, Shanghai, China; aThese authors contributed equally
}

\section{Key Words}

Shear stress • Endothelial cells • Proliferation • Vascular smooth muscle cells • SIRT1 • Connexin40

\begin{abstract}
Background: Shear stress imposed by blood flow directly impacts endothelial cells (ECs), which are simultaneously influenced by neighboring vascular smooth muscle cells (VSMCs). However, the mechanisms by which shear stress and VSMCs modulate EC proliferation remain to be elucidated. Methods: ECs, cultured alone or co-cultured with VSMCs, were subjected to a normal level of laminar shear stress (NSS) of $15 \mathrm{dyne} / \mathrm{cm}^{2}$ or kept under static conditions by using a parallel-plate flow chamber system, respectively. Results: BrdU incorporation assay and flow cytometry revealed that NSS inhibited EC proliferation with or without VSMCs. Western blot analysis demonstrated that NSS down-regulated the expression of Connexin40 (Cx40) in both ECs cultured alone and ECs co-cultured with VSMCs, accompanied by the increased expression of SIRT1. Moreover, salermide, an inhibitor of SIRT1, as well as SIRT1-specific siRNA transfection inhibited the effect of NSS on EC proliferation and Cx40 expression. In contrast, resveratrol, a SIRT1 activator, induced an alteration in ECs similar to the application of NSS. Conclusion: NSS inhibits the proliferation of ECs via SIRT1 and Cx40 in the presence or absence of VSMCs. The data suggest that NSS plays a protective role in vascular homeostasis by maintaining EC proliferation at a normal level.
\end{abstract}




\section{Introduction}

Vascular endothelial cells (ECs), which form the interface between the flowing blood and the vessel wall, are exposed to mechanical forces induced by the pulsatile blood flow, such as shear stress, circumferential stretch and hydrostatic pressure. ECs are uniquely exposed to shear stress at the luminal surface of the blood vessel, and flow shear stress is one of the mechanical stimuli that regulate the balance of protein synthesis, morphology, migration, proliferation and survival of ECs [1]. Many studies have shown the effect of shear stress on EC proliferation. For example, steady laminar shear stress, as an atheroprotective factor, inhibits EC proliferation [2-5]. However, the majority of these studies used a monolayer of ECs cultured alone as an experimental model, which may not accurately reflect the environment of ECs in vivo. ECs are also influenced by neighboring vascular smooth muscle cells (VSMCs) in the blood vessel wall. The interaction between ECs and VSMCs plays an important role in maintaining the normal vascular structure and function [6-9]. However, the effect of shear stress on EC proliferation in presence of VSMCs and the underlying mechanisms involved have not been well elucidated.

There are reports of the transmission of mechanical signals through gap junction proteins, which consist of intercellular channels that ensure direct communication between ECs and VSMCs [10]. Gap junctions (GJs) are channel-like proteins that connect the cytoplasm of neighboring cells and allow small molecules and ions to pass freely between the cells, modulating and synchronizing their intracellular environments [11]. Studies have shown that ECs express 3 types of GJ proteins called connexins, Cx37, Cx40 and Cx43 $[8,12]$, and VSMCs typically express Cx37, Cx40, Cx43 and Cx45. Cx40 is located predominantly in the ECs, and $\mathrm{Cx} 43$ is the most prominent gap-junction protein found in the VSMCs [13]. Mutations in the endothelial connexin genes are associated with atherosclerosis, cardiac infarction, and hypertension. Both Cx40 and Cx43 participate in ballooninduced neointimal proliferation [14]. While, Li, et al. reported that $\mathrm{Cx} 43$, rather than $\mathrm{Cx} 40$, contributes to the proliferation of VSMCs suppressed by the angiotensin II (Ang II)converting enzyme inhibitors in balloon injury [15]. Since there is evidence that mechanical stress modulates the expression of $\mathrm{Cx} 43$ [16], we hypothesized that connexins are involved in the modulation of EC proliferation by shear stress and sought to determine what type(s) of connexins were involved.

SIRT1, also known as Sirtuin 1, is a nicotinamide adenine dinucleotide (NAD)-dependent class III histone deacetylase (HDAC). SIRT1 removes acetyl groups from many non-histone proteins, and is involved in a broad range of physiological functions, including the control of gene expression, metabolism, and aging $[17,18]$. Increasing evidence has demonstrated the protective roles for SIRT1 in vascular biology and atherosclerosis. SIRT1 prevents hyperglycemia-induced endothelial cell dysfunction by repressing p66Shc expression [19]. SIRT1 has been found to modulate proliferation and senescence in ECs [20, 21]. A recent study revealed that shear stress regulates SIRT1 expression and activity [22]. Furthermore, trichostatin A, an inhibitor of HDAC I/II, was found to up-regulate the expression of $\mathrm{Cx} 43$ in prostate cancer cells and hepatocytes and down-regulate the expression of Cx26 in hepatocytes [23-25]. However, the relationship between SIRT1 and connexins remains unknown. Therefore, we further analyzed whether SIRT1 and connexins were involved in the modulation of EC proliferation by shear stress.

Our studies were designed to determine the effect of NSS on the proliferation of ECs cultured alone or co-cultured with VSMCs and the roles of connexins and SIRT1 in the process. These data will provide important insights into the mechanisms whereby shear stress regulates EC functions. 


\section{Materials and Methods}

\section{Cell culture}

The animal care and experimental protocols conformed to the Animal Management Rules of China (Documentation 55, 2001, Ministry of Health, China). ECs and VSMCs were isolated from the thoracic aortas of male Sprague-Dawley rats, 220-260 g, by a digestive method for ECs [26] and an explant method for VSMCs as previously described [27]. ECs, between passages 2 and 4, and VSMCs, between passages 4 and 8, with cell populations of more than $95 \%$ purity were used in all experiments.

\section{EC-VSMC co-culture model and shear stress loading}

A co-culture model was established using a cell culture insert with a $10 \mu \mathrm{m}$ thick porous polyethylene terephthalate (PET) membrane (Becton Dickinson Labware, NJ, USA) as previously described [28]. Briefly, each PET membrane contains $1.6 \times 10^{6}$ pores $/ \mathrm{cm}^{2}$, and each pore is $0.4 \mu \mathrm{m}$ in diameter. ECs were seeded onto the outer surface of the PET membrane at a density of $2 \times 10^{5}$ cells per insert. After incubating for 8 hours to allow for attachment, the insert was placed with the EC side down into a 6-well plate containing complete culture medium. For the EC-VSMC co-culture model, VSMCs were seeded onto the inner surface of the PET membrane at a density of $1-2 \times 10^{5}$ cells per insert. ECs were maintained in Medium 199 (Gibco) essential medium supplemented with $20 \%$ heat-inactivated fetal bovine serum (Gibco), $5 \mathrm{ng} / \mathrm{ml}$ human aFGF (Sigma), $100 \mathrm{mmol} / \mathrm{L}$ HEPES, $100 \mathrm{U} / \mathrm{mL}$ heparin (Sigma), $100 \mathrm{U} / \mathrm{mL}$ penicillin and $100 \mu \mathrm{g} / \mathrm{mL}$ streptomycin. VSMCs were maintained in Dulbecco's modified Eagle's medium (DMEM, Gibco) containing 10\% heat-inactivated fetal bovine serum, $100 \mathrm{U} / \mathrm{mL}$ penicillin and $100 \mu \mathrm{g} / \mathrm{mL}$ streptomycin. When the cells reached confluence, the insert, which had been seeded with ECs alone or co-cultured with VSMCs, was assembled into a parallelplate flow chamber system as previously described [26], and a normal level of laminar shear stress (NSS) of 15 dyne $/ \mathrm{cm}^{2}$ was applied to the ECs for 12 hours.

There were 4 groups in the present study: (a) ECs cultured alone in the static mode (the control, EC/O); (b) ECs cultured alone and exposed to NSS for 12 hours (EC/O+NSS); (c) ECs co-cultured with VSMCs in the static mode (EC/VSMC); and (d) ECs co-cultured with VSMCs and exposed to NSS for 12 hours (EC/ VSMC+NSS).

\section{Cell proliferation assays}

The cell proliferation was analyzed with a colorimetric bromodeoxyuridine (BrdU) kit (Roche Diagnostics) and flow cytometry (FCM). For the BrdU ELISA, after the application of NSS, the cells were digested and seeded at a density of $1 \times 10^{5}$ cells per well in 96-well plates, the BrdU labeling reagent was added into the culture medium (1:1000), and then the plates were incubated for 12 hours. The cells were fixed with a FixDenat solution for 30 minutes at room temperature and then incubated with an anti-BrdU peroxidase working solution (freshly diluted 1:100) for 90 minutes according to the manufacturer's instructions. Following three rinses with washing buffer, $100 \mu \mathrm{l} /$ well of substrate solution was added to the cells and incubated for 20 minutes at room temperature. Thereafter, $25 \mu \mathrm{H} \mathrm{H}_{2} \mathrm{SO}_{4}(1 \mathrm{~mol} / \mathrm{L})$ was added to each well, and the 96-well plates were shaken for 1 minute. The absorbance at $450 \mathrm{~nm}$ was measured in an ELISA plate reader (Bio-Rad 680).

For the FCM assay, after NSS application, the cells were treated with a $0.125 \%$ trypsin solution, gently fixed by adding $75 \%$ ethanol, and placed in a $-20^{\circ} \mathrm{C}$ freezer for 2 hours. The cells were then treated with $0.5 \%$ Triton X-100 for 5 minutes in an ice bath. The cells were resuspended in $300 \mu \mathrm{L}$ of PBS containing 50 $\mu \mathrm{g} / \mathrm{mL}$ propidium iodide and $10 \mu \mathrm{g} / \mathrm{mL}$ RNase. The cells were then incubated in a dark room for 30 minutes at room temperature and analyzed with a FACScan flow cytometer (BD Biosciences FACSCalibur ${ }^{\mathrm{TM}}$ ).

\section{SDS-PAGE and Western blot analysis}

Protein extracts were separated by $12 \%$ SDS-PAGE. The proteins were detected using primary antibodies specific for Cx37 (Abcam, 1:500), Cx40 (Santa Cruz Biotechnology, 1:600), Cx43 (Zymed, 1:600), SIRT1 (Santa Cruz Biotechnology, 1:300), and GAPDH (Santa Cruz Biotechnology, 1:600). After incubation with alkaline phosphatase-conjugated secondary antibodies (Jackson Immunoresearch), the signals were visualized by nitroblue tetrazolium- bromochloroindolyl phosphate (Bio Basic, Inc.) and quantified with Quantity One software (Bio-Rad). 
Inhibitor and activator protocols and SIRT1 SiRNA transfection

For the inhibitor and activator studies, resveratrol and salermide, a specific pharmacological activator and inhibitor of SIRT1, respectively, were used. Alcohol and DMSO, the respective solvent for resveratrol and salermide, was present at same concentrations in all groups, including the control. ECs were treated with resveratrol $(50 \mu \mathrm{mol} / \mathrm{L}$, Sigma) for 24 hours in the static mode, or pretreated with salermide (50 $\mu \mathrm{mol} / \mathrm{L}$, Merck) for 12 hours prior to NSS loading. Controls were included in each case.

For the RNA interference experiment, ECs were transfected with $100 \mathrm{nmol} / \mathrm{L}$ of SIRT1 siRNA or control non-silencing siRNA (Gene-Pharma, China) for 6 hours using lipofectamine 2000 (Invitrogen) in Opti-MEM medium (Gibco) according to the manufacturer's instructions. The sequences of the siRNA oligos are as follows:

SIRT1 siRNA: sense 5'-GAU UUA UUA CCA GAA ACA ATT-3' and anti-sense 5'-UUG UUU CUG GUA AUA AAU CTT-3'; Cx40 siRNA: sense 5'-GGG UAC UGC AGA UCA UCU UTT-3' and anti-sense 5'-AAG AUG AUC UGC AGU ACC CTT-3'; Cx43 siRNA: sense 5'-GAU UUA UUA CCA GAA ACA ATT-3' and anti-sense 5'-UUG UUU CUG GUA AUA AAU CTT-3'; non-silencing siRNA: sense 5'-UUC UCC GAA CGU GUC ACG UTT-3' and anti-sense 5'ACG UGA CAC GUU CGG AGA ATT -3'.

\section{Immunofluorescence staining}

The attached ECs were fixed in 3.7\% formaldehyde for 20 minutes, permeabilized for 5 minutes in $0.5 \%$ Triton X-100, and blocked with $10 \%$ normal fetal bovine serum in PBS for 30 minutes. Then, cells were incubated with the primary antibody against $\mathrm{Cx} 40$ (Santa Cruz Biotechnology, 1:25) at $4{ }^{\circ} \mathrm{C}$ over night. ECs incubated without primary antibody served as the negative control. All the samples were washed in PBS for 30 minutes and incubated with a FITC-conjugated secondary antibody (Jackson Immunoresearch) along with DAPI for 1 hour at room temperature. The samples were examined with a laser scanning confocal microscope (Olympus, LV1000).

\section{Statistical analysis}

Each experiment was performed at least in triplicate, and all values are expressed as the mean $\pm S D$. The student's $t$-test was used to compare two groups and the multiple comparisons were performed by one way ANOVA. Values of $p<0.05$ were accepted as statistically significant, and $p<0.01$ was considered to be remarkably significant.

\section{Results}

The effects of NSS and VSMCS on EC proliferation

ECs cultured alone or co-cultured with VSMCs were either exposed to $15 \mathrm{dyne} / \mathrm{cm}^{2}$ of NSS or kept under static conditions for 12 hours. EC proliferation was evaluated by measuring BrdU incorporation into newly synthesized DNA, as well as the cell cycle distribution by FCM. As shown in Fig. 1A, the proliferation of ECs subjected to NSS was less than that in the static cultures both for ECs cultured alone and co-cultured with VSMCs. Fig. 1B shows that NSS also significantly reduced the percentage of cells in S phase in EC/O+NSS $(1.92 \pm 0.15$ $\%)$ VS. EC/O (4.21 $\pm 0.31 \%)$, and EC/VSMC+NSS (2.44 $\pm 0.27 \%)$ VS. EC/VSMC $(8.43 \pm 0.23$ $\%)$. There was no significant difference in the percentage of cells in G1/G0 and G2/M among all the groups. Under the static condition, the co-cultured VSMCs increased EC proliferation (Fig. 1A) and the percentage of ECs in S phase (Fig. 1B, EC/VSMC $8.43 \pm 0.23 \%$ vs. EC/O $4.21 \pm 0.31 \%$ ) compared with ECs cultured alone. The results clearly demonstrate that NSS inhibits the proliferation of ECs either with or without VSMCs. Although VSMCs enhance EC proliferation, the application of NSS attenuates the effect of VSMCs on EC proliferation.

\section{NSS modulates the expression of SIRT1 and connexins in ECS}

The effect of NSS on SIRT1 and connexin expression was determined by Western blot analysis after NSS application to the ECs for 12 hours. As shown in Fig. 2, NSS increased SIRT1 expression and decreased Cx40 expression in groups EC/O+NSS and EC/VSMC+NSS compared with groups EC/O and EC/VSMC, respectively. However, there was no significant 
Fig. 1. The effects of NSS and VSMCs on EC proliferation. A. The proliferation of ECs was determined by a colorimetric BrdU kit. B. The proliferation of ECs was determined by FCM. The four columns from left to right are EC/O (ECs cultured alone in the static mode); EC/O+NSS (ECs cultured alone with NSS); EC/VSMC (ECs cocultured with VSMCs in the static mode); EC/VSMC+NSS (ECs cocultured with VSMCs and exposed to NSS). Values shown are the mean $\pm S D$ for each group from at least three independent experiments, ${ }^{*} p<0.05,{ }^{* *} p<0.01, n=5$.

Fig. 2. The effect of NSS on the expression of SIRT1 and Connexins. The four columns from left to right are EC/O (ECs cultured alone in the static mode); EC/O+NSS (ECs cultured alone with NSS); EC/VSMC (ECs co-cultured with VSMCs in the static mode); and EC/VSMC+NSS (ECs co-cultured with VSMCs and exposed to NSS). The top panel represents the Western blot results. Values shown are the mean $\pm S D$ for each group from at least three independent experiments, ${ }^{*} p<0.05,{ }^{* *} p<0.01, n=5$.

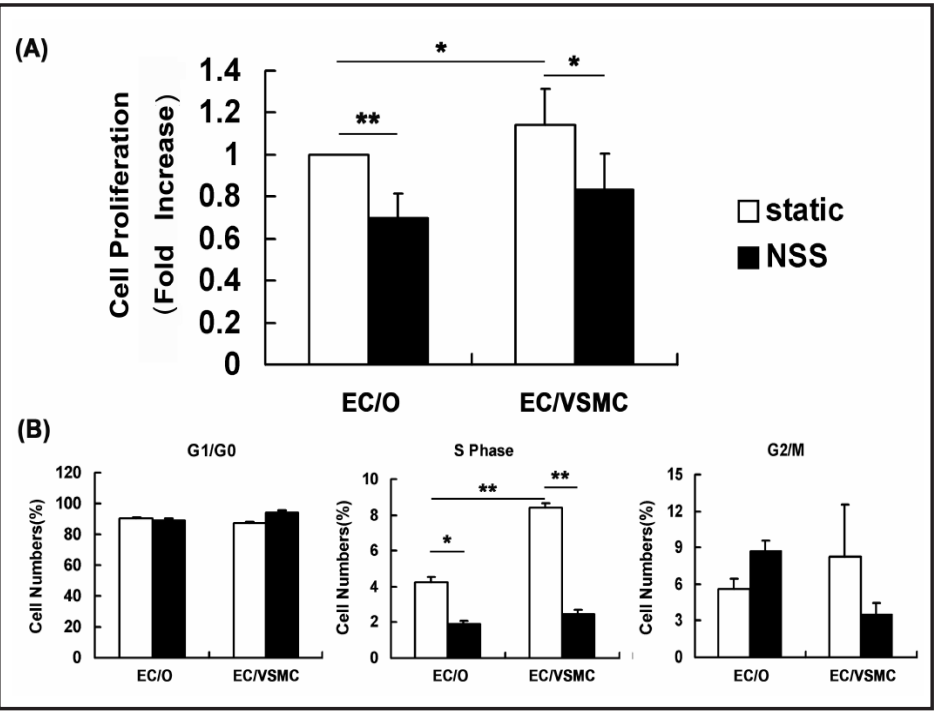

difference between groups EC/VSMC and EC/O. Fig. 2 also shows that NSS decreased Cx43 expression in ECs cultured alone, but there was no effect in ECs co-cultured with VSMCs. However, in the static mode, VSMCs decrease Cx43 expression compared with ECs cultured alone. There was no statistically significant difference in Cx37 expression among all the groups. These results indicate that in the co-cultured model SIRT1 and Cx40 are sensitive to NSS and may be involved in the NSS-modulated proliferation of ECs, while Cx37 and Cx43 may be not.

Silencing SIRT1 and CX40 increased proliferation of ECs cultured alone in the static mode

Fig. 3 indicates that silencing SIRT1 increased Cx40 expression and EC proliferation. However, "knocking down" Cx40 attenuated SIRT1 expression and increased EC proliferation. $\mathrm{Cx} 43$ did not appear to be involved in the process. The results demonstrate that SIRT1 and Cx40 can influence each other. SIRT1 negatively regulates the expression of Cx40 in ECs, 
Fig. 3. The effect of silencing SIRT1 and Cx40 on EC proliferation. A. The proliferation of ECs was determined by a colorimetric BrdU kit. ECs were cultured alone in the static mode and treated for 36 hours as follows: transfected with a non-silencing siRNA as a control, transfected with the SIRT1 siRNA $(100 \mathrm{nmol} / \mathrm{mL})$, the Cx40 siRNA $(100 \mathrm{nmol} /$ $\mathrm{mL}$ ) or the Cx43 siRNA (100 nmol/mL). B. The top panel represents the Western blot results. Values shown are the mean $\pm S D$ for each group from at least three independent experiments, ${ }^{*} p<0.05, n=5$.

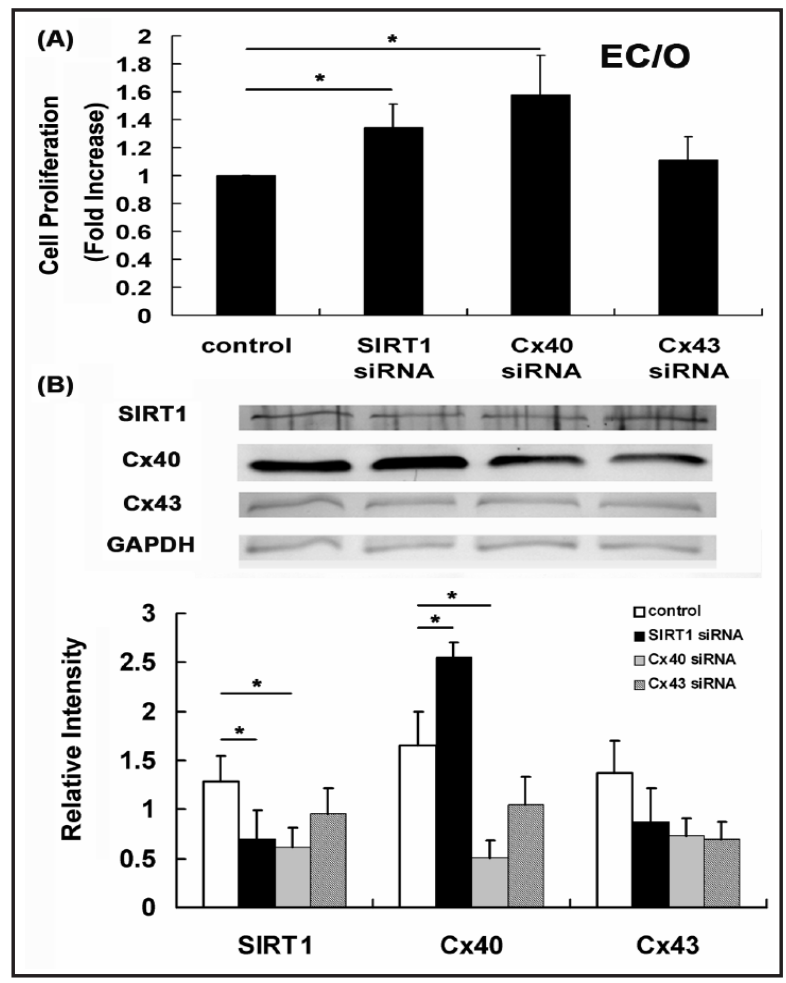

Fig. 4. The modulation of $\mathrm{Cx} 40$ distribution by SIRT1. SIRT1 activation by resveratrol downregulated the expression of $\mathrm{Cx} 40$. However, inhibition of SIRT1 activity by salermide or the siRNA specific for SIRT1 up-regulated the expression of $\mathrm{Cx} 40$ in ECs. ECs were cultured alone in the static mode and treated as indicated for 24 hours with the inhibitor and the same amount of solvent as the control: A. Alcohol, solvent of resveratrol; B. resveratrol (50 $\mu \mathrm{mol} / \mathrm{L})$, an activator of SIRT1; C. DMSO, solvent of salermide; D. salermide $(50 \mu \mathrm{mol} / \mathrm{L})$, an inhibitor of SIRT1; E. transfected with non-silencing siRNA; F. transfected with SIRT1 siRNA (100 nmol/mL). ECs were incubated with the primary antibody against Cx40 and incubated with a FITC-conjugated secondary antibody along with DAPI. The samples were examined with a laser scanning confocal microscope (Olympus, LV1000). Bar $=30 \mu \mathrm{m}, n=3$.

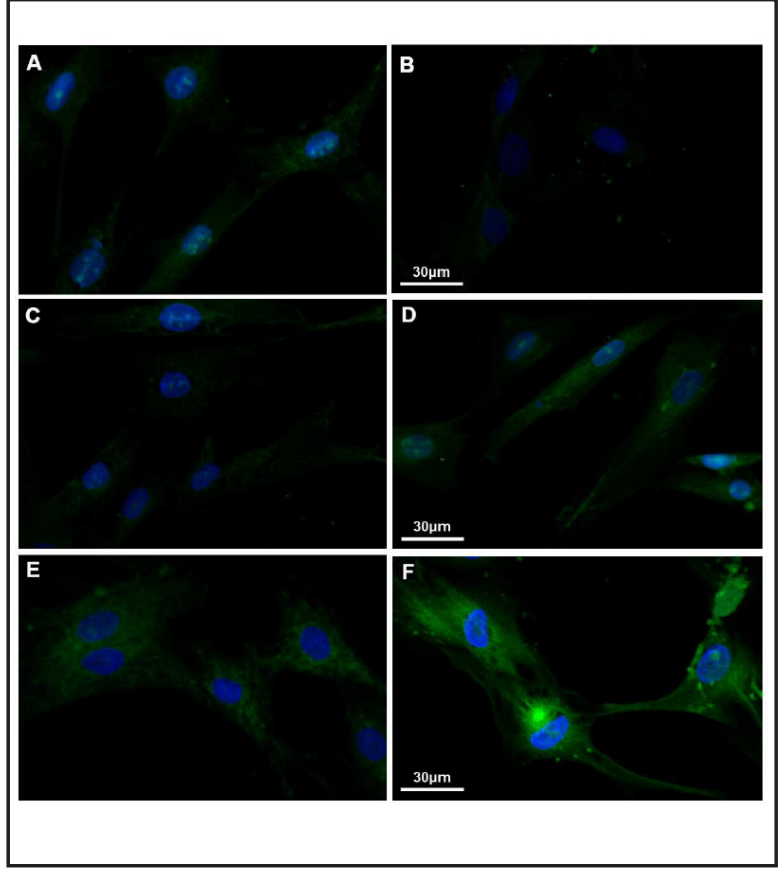

and the decreased Cx40 also down-regulates the expression of SIRT1. The down-regulation of SIRT1 enhances EC proliferation. Therefore, SIRT1 plays a critical role in modulating EC proliferation.

\section{Immunostaining of Cx40 in ECs cultured alone in the static mode}

Cx40 distribution, regulated by SIRT1, in ECs was examined by immunostaining. ECs were cultured alone and treated with resveratrol (Fig. 4B) or salermide (Fig. 4D) for 12 hours. The images of the immunostained cells indicate that salermide increased while resveratrol 


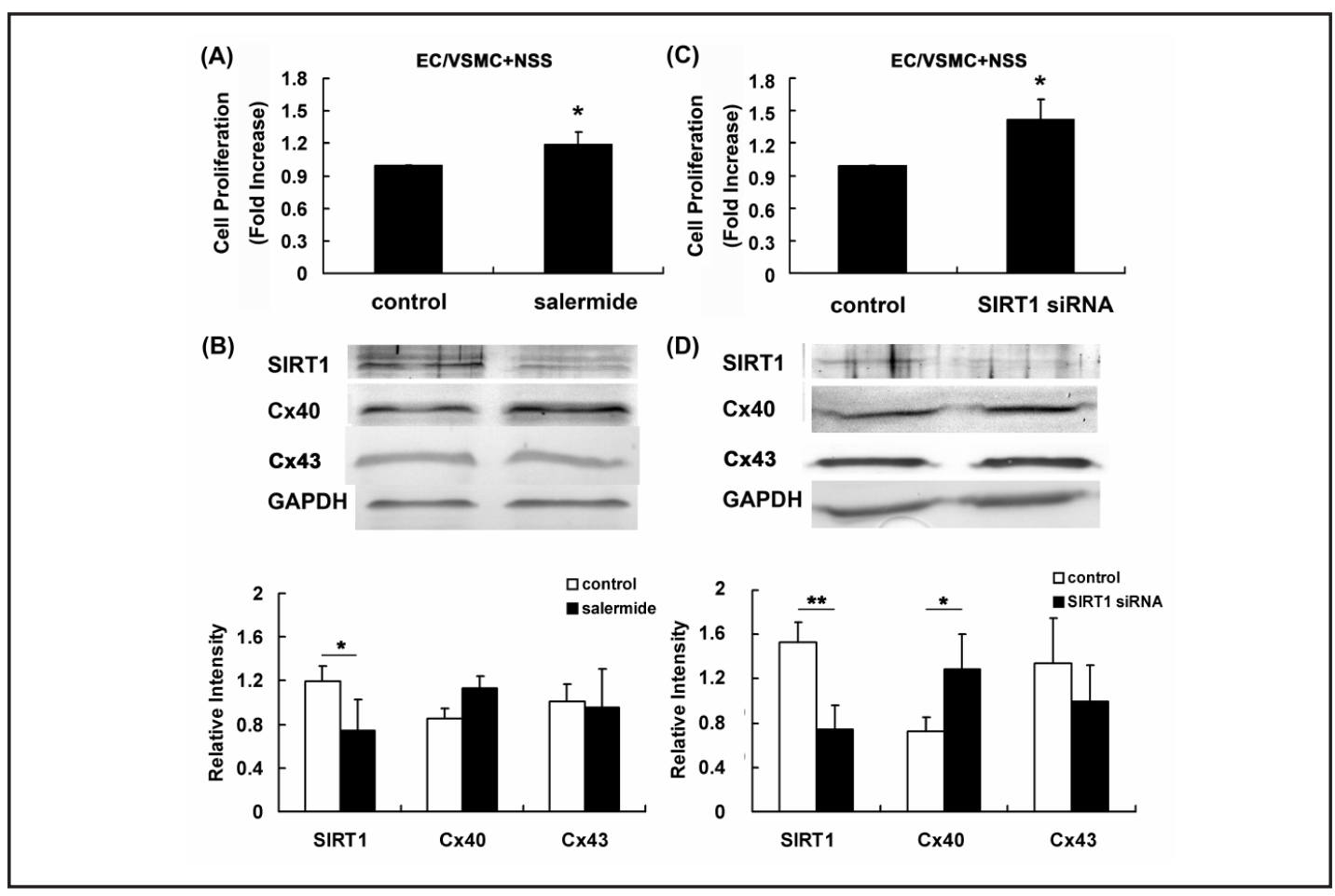

Fig. 5. The top histogram shows that salermide, an inhibitor of SIRT1 (A), and the siRNA specific for SIRT1 (B) induced the proliferation of ECs co-cultured with VSMCs and exposed to NSS for 12 hours. Quiescent ECs were pretreated with salermide $(50 \mu \mathrm{mol} / \mathrm{L})$ for 12 hours (the control was pretreated with DMSO), or transfected with control siRNA or the siRNA specific for SIRT1 (100 nmol/mL) for 24 hours. EC proliferation was determined by a colorimetric BrdU kit. The bottom graph demonstrates that both salermide (C) and the SIRT1 siRNA (D) decreased SIRT1 and increased Cx40. The treatments are as indicated in (A) and (B). The panels represent the Western blot results. Values shown are the mean $\pm S D$ for each group from at least three independent experiments, ${ }^{*} p<0.05,{ }^{* *} p<0.01$ vs. the control, $n=5$.

decreased the expression of $\mathrm{Cx} 40$ compared with their respective control (Fig. $4 \mathrm{~A}$ and $\mathrm{C}$ ). Furthermore, the ECs transfected with the siRNA of SIRT1 expressed more Cx40 than those transfected with the non-silencing siRNA (Fig. 4E and F). These results further confirm that SIRT1 is responsible for modulation of Cx40 expression in ECs.

Silencing SIRT1 inhibited the NSS-induced modulation of EC proliferation via CX40 in coculture model

Whether SIRT1 was responsible for the NSS-mediated modulation of EC proliferation was examined by using a SIRT1 pharmacological inhibitor, salermide, and SIRT1-specific siRNA transfection. The co-cultured ECs were treated with salermide for 12 hours or transfected with the SIRT1 siRNA for 24 hours prior to loading NSS. As shown in Fig. 5A and $B$, salermide resulted in an increase in the proliferation of ECs and a significant decrease in SIRT1 expression. Although the expression of $\mathrm{Cx} 40$ seemed to be higher than the control, the difference was not statistically significant (Fig. 5B). Using the siRNA specific for SIRT1 resulted in a significant decrease in SIRT1 expression and an increase in Cx40 expression as well as the proliferation of ECs (Fig. 5C and D). The SIRT1 siRNA inhibited more efficiently than salermide did. These results indicate that inhibiting SIRT1 can reverse the effect of NSS by inducing Cx40 expression and EC proliferation and strongly suggest that SIRT1 plays a pivotal role in the process whereby NSS modulates EC proliferation and Cx40 expression. 
Fig. 6. Resveratrol modulates EC proliferation and the expression of Cx40. The top histogram (A) indicates that resveratrol, an activator of SIRT1, inhibited the proliferation of ECs co-cultured with VSMCs in the static mode. ECs co-cultured with VSMCs were treated with resveratrol $(50 \mu \mathrm{mol} / \mathrm{L})$ for 24 hours and analyzed with a colorimetric BrdU kit. The bottom graph (B) indicates that resveratrol increased SIRT1 and decreased Cx40. The treatments were as described in (A) and the panels represent the Western blot results. Values shown are the mean $\pm S D$ for each group from at least three independent experiments, ${ }^{*} p<0.05 v s$. the control, $n=8$.

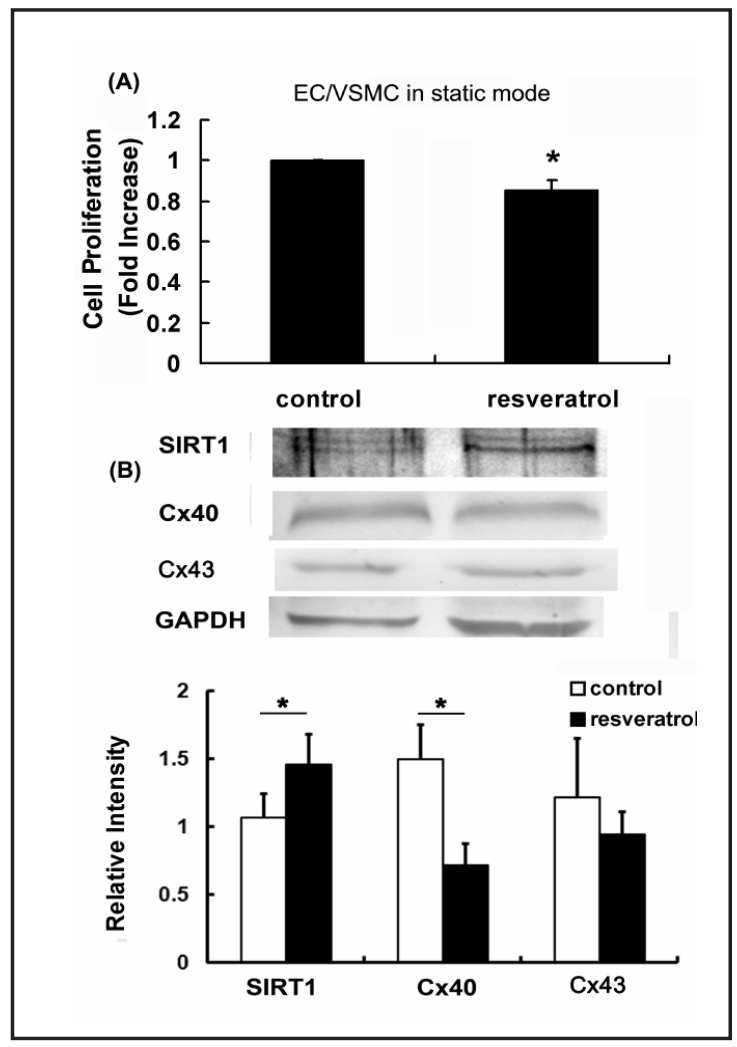

Resveratrol, a SIRT1 activator, has effects on EC proliferation and the expression of CX40 similar to NSS

To investigate whether SIRT1 activity directly modulates EC proliferation and Cx40 expression, the co-cultured ECs under static conditions were incubated with resveratrol, a SIRT1 activator. We found that resveratrol down-regulated EC proliferation and decreased Cx40 expression (Fig. 6A and B), which were similar to the effects of NSS loading. These results demonstrate that the down-regulation of EC proliferation and decreased expression of $\mathrm{Cx} 40$ are induced by SIRT1, which is activated by either NSS or a specific pharmacological activator.

\section{Discussion}

ECs exposed to shear stress undergo a series of modifications in gene expression, which lead to functional changes. However, most of these prior studies did not consider the influence of neighboring VSMCs on ECs. Accumulating evidence has demonstrated that ECs and VSMCs affect each other in blood vessels [9, 28-31]. One of the previous studies from our lab has shown that either NSS or VSMCs enhance EC migration separately, but the presence of NSS inactivates the effect of VSMCs on EC migration. In other words, NSS may play a protective role in vascular homeostasis by modulating EC migration [29]. However, the effects of NSS on EC proliferation in the presence of VSMCs and the underlying mechanisms involved remain unclear. The present study demonstrates that NSS inhibits the proliferation of ECs either alone or co-cultured with VSMCs via SIRT1 and the Cx40 gap junction channel, and the results suggest that NSS exerts a protective influence on vascular homeostasis by modulating EC proliferation to a normal level.

Other investigators have shown that mechanical signals are transmitted through gap junction proteins, which consist of intercellular channels that ensure direct interaction between ECs and VSMCs and the synchronization of their behavior along the vascular tree [10- 
11]. Physiological variations in the expression of connexins are observed along the vascular tree. There are many different reports concerning the function of connexins using different models, e.g., deletion of the $\mathrm{Cx} 40$ gene in mice results in a marked sustained hypertension [32]. In an in vitro study, $\mathrm{Cx} 43$ can be increased by disturbed flow [33], and an in vivo experiment has shown that Cx43 is highly localized to sites of disturbed flow in rat aortic ECs, but Cx37 and $\mathrm{Cx} 40$ are more uniformly distributed in situ [34]. When NSS provides a stimulatory signal, it is unclear from the existing evidence which vascular connexin isoforms, such as $\mathrm{Cx} 37, \mathrm{Cx} 40$ or $\mathrm{Cx} 43$, are involved and which gap junction locations are targeted, especially in the case where NSS affects the proliferation of ECs cultured with or without VSMCs. In the current study, an EC-VSMC co-culture system was used to mimic the physiological condition. Our results reveal that when ECs are cultured either with or without VSMCs, NSS represses EC proliferation accompanied by the down-regulation of $\mathrm{Cx} 40$ and up-regulation of SIRT1. However, Cx43 levels are down regulated in the EC by NSS, the co-cultured cells show a lower level of $\mathrm{Cx} 43$ which is not affected by NSS, which might be caused by both NSS and co-culture with VSMCs reduced the expression of Cx43 in EC compared with EC cultured along in static, and the existence of physiological component including VSMC and NSS played a notable role in reducing $\mathrm{Cx} 43$ to a low level without duplicate effect. Therefore, we first obtained quantifiable data to confirm that physiological steady shear stress modulates the expression of $\mathrm{Cx} 40$ and, to a lesser extent, Cx37 and Cx43 in ECs in the co-culture model system. This experimental model more closely approaches the physiological condition than a simple monolayer culture.

SIRT1 has long been considered to be an anti-aging target and plays a critical role in prevention of cell senescence. The over-expression of SIRT1 inhibits prostate cancer cell lines [35] and pancreatic cancer cells [36]. While Zu, et al. reported SIRT1 promotes proliferation of primary porcine aortic ECs measured by crystal violet [21], our results showed that the down-regulation of rat aortic EC proliferation is induced when SIRT1 is activated by either NSS or resveratrol, which is an activator doubled the rate of deacetylation by SIRT1 at about $11 \mu \mathrm{M}$ [37]. Resveratrol increased the proliferative, migratory, and adhesive activities of endothelial progenitor cells [38]. A recent study showed that resveratrol inhibited cell proliferation in a time- and dose-dependent manner in cultured human aortic and pulmonary arterial ECs [39]. Cell number of human umbilical vein ECs in culture is decreased drastically at higher concentration of resveratrol $(10$ and $100 \mathrm{mg} / \mathrm{ml})$. Proliferation promoting effect or no effect was also observed if the amount of resveratrol applied was very little [40-41]. Therefore, these contrary results may be due to differences in sensitivity of resveratrol among these cells, the dose of resveratrol and the methods for examining cell proliferation.

The list of SIRT1 substrates is continuously growing and includes several transcription factors, such as the tumor suppressor protein p53, members of the FOXO family (Forkhead box factors regulated by insulin/Akt), and NFKB (nuclear factor kappa B) [42]. Chen, et al reported that laminar shear stress is atheroprotective and modulates eNOS via AMPK and SIRT1 [22]. Moreover, Cx40 and eNOS may form a protein complex, as evidenced by both immunoprecipitation and an in situ proximity ligation assay [43]. In the future, the relationship between SIRT1, Akt, eNOS and Cx40 should be investigated.

This study provides novel results regarding the relationship between the expression of Cx40, SIRT1, and EC proliferation under NSS in the co-culture model system and demonstrates that steady laminar shear stress inhibits the proliferation of ECs either with or without VSMCs via SIRT1 and Cx40. These findings may provide new insights into the regulation of EC biology by shear stress.

\section{Acknowledgements}

This research was supported by grants from the National Natural Science Foundation of China, Nos. 10772120, 10972141, 11202133. 
Yao et al.: NSS Inhibits Proliferation of ECs Co-Cultured with VSMCs

\section{References}

1 Kadohama T, Nishimura K, Hoshino Y, Sasajima T, Sumpio BE: Effects of different types of fluid shear stress on endothelial cell proliferation and survival. J Cell Physiol 2007;212:244-251.

2 Levesque MJ, Nerem RM, Sprague EA: Vascular endothelial cell proliferation in culture and the influence of flow. Biomaterials 1990;11:702-707.

3 Dimmeler S, Haendeler J, Rippmann V, Nehls M, Zeiher AM: Shear stress inhibits apoptosis of human endothelial cells. FEBS Lett 1996;399:71-74.

4 Lehoux S, Tedgui A: Signal transduction of mechanical stresses in the vascular wall. Hypertension 1998;32:338-345.

-5 Lin K, Hsu PP, Chen BP, Yuan S, Usami S, Shyy JY, Li YS, Chien S: Molecular mechanism of endothelial growth arrest by laminar shear stress. Proc Natl Acad Sci USA 2000;97:9385-9389.

6 Tsai MC, Chen L, Zhou J, Tang Z, Hsu TF, Wang Y, Shih YT, Peng HH, Wang N, Guan Y, Chien S, Chiu JJ: Shear stress induces synthetic-to- contractile phenotypic modulation in smooth muscle cells via peroxisome proliferator-activated receptor alpha/delta activations by prostacyclin released by sheared endothelial cells. Circ Res 2009;105:471-480.

7 Harvey KA, Welch Z, Sliva D, Siddiqui RA: Role of Rho kinase in sphingosine 1-phosphate-mediated endothelial and smooth muscle cell migration and differentiation. Mol Cell Biochem 2010;342:7-19.

8 Wallace CS, Truskey GA: Direct-contact co-culture between smooth muscle and endothelial cells inhibits TNF-alpha-mediated endothelial cell activation. Am J Physiol Heart Circ Physiol 2010;299:H338-346.

-9 Qi YX, Jiang J, Jiang XH, Wang XD, Ji SY, Han Y, Long DK, Shen BR, Yan ZQ Chien S, Jiang ZL: PDGF-BB and TGF-(beta)1 on cross-talk between endothelial and smooth muscle cells in vascular remodeling induced by low shear stress. Proc Natl Acad Sci USA 2011;108:1908-1913.

10 Johnstone S, Isakson, B, Locke D: Biological and biophysical properties of vascular connexin channels. Int Rev Cell Mol Bio 2009;278:69-118.

11 Figueroa XF, Duling BR: Gap junctions in the control of vascular function. Antioxid Redox Sign 2009;11:251-266.

12 Zhou B, Margariti A, Zeng L, Xu Q: Role of histone deacetylases in vascular cell homeostasis and arteriosclerosis. Cardiovas Res 2011;90:413-420.

13 Van Kempen MJ, Jongsma HJ: Distribution of connexin37, connexin40 and connexin43 in the aorta and coronary artery of several mammals. Histochem Cell Biol 1999;112:479-486.

14 Wang LH, Chen JZ, Sun YL, Zhang FR, Zhu JH, Hu SJ, Wang DH: Regulation of connexin expression after ballon injury: possible mechanisms for antiprolifirative effect of statins. Am J Hypertens 2005;18:11461153.

15 Li DQ, Chen WX, Zhou YP, Han Y: Effect of ramipril on the regulation of the expression of connexins 40 and 43 in a ribbit model of arterial balloon injury. Mol Med Rep 2012;6:565-569.

16 Cowan DB, Lye SJ, Langille BL: Regulation of vascular connexin43 gene expression by mechanical loads. Circ Res 1998;82:786-793.

17 Haigis MC, Guarente LP: Mammalian sirtuins--emerging roles in physiology, aging, and calorie restriction. Genes Dev 2006;20:2913-2921.

18 Yamamoto H, Schoonjans K, Auwerx J: Sirtuin functions in health and disease. Mol Endocrinol 2007;21:1745-1755.

19 Zhou S, Chen HZ, Wan YZ, Zhang QJ, Wei YS, Huang S, Liu JJ, Lu YB, Zhang ZQ, Yang RF, Zhang R, Cai H, Liu DP, Liang CC: Repression of P66Shc Expression by SIRT1 Contributes to the Prevention of HyperglycemiaInduced Endothelial Dysfunction. Circ Res 2011;109:639-648.

20 Ota H, Eto M, Kano MR, Kahyo T, Setou M, Ogawa S, Iijima K, Akishita M, Ouchi Y: Induction of endothelial nitric oxide synthase, SIRT1, and catalase by statins inhibits endothelial senescence through the Akt pathway. Arterioscler Throm Vasa Biol 2010;30:2205-2211.

-21 Zu Y, Liu L, Lee MY, Xu C, Liang Y, Man RY, Vanhoutte PM, Wang Y: SIRT1 promotes proliferation and prevents senescence through targeting LKB1 in primary porcine aortic endothelial cells. Circ Res 2010;106:1384-1393.

22 Chen Z, Peng IC, Cui X, Li YS, Chien S, Shyy JY: Shear stress, SIRT1, and vascular homeostasis. Proc Natl Acad Sci USA 2010;107:10268-10273.

23 Henkens T, Vinken M, Lukaszuk A, Tourwe D, Vanhaecke T, Rogiers V: Differential effects of hydroxamate histone deacetylase inhibitors on cellular functionality and gap junctions in primary cultures of mitogenstimulated hepatocytes. Toxicol Lett 2008;178:37-43. 
Yao et al.: NSS Inhibits Proliferation of ECs Co-Cultured with VSMCs

24 Jung JW, Cho SD, Ahn NS, Yang SR, Park JS, Jo EH, Hwang JW, Aruoma OI, Lee YS, Kang KS: Effects of the histone deacetylases inhibitors sodium butyrate and trichostatin A on the inhibition of gap junctional intercellular communication by $\mathrm{H}_{2} \mathrm{O}_{2}$ - and 12-0-tetradecanoylphorbol-13-acetate in rat liver epithelial cells. Cancer Lett 2006;241:301-308.

25 Henkens T, Vinken M, Lukaszuk A, Tourwe D, Vanhaecke T, Rogiers V: Differential effects of hydroxamate histone deacetylase inhibitors on cellular functionality and gap junctions in primary cultures of mitogenstimulated hepatocytes. Toxicol Lett 2008;178:37-43.

26 Kwan HY, Leung PC, Huang Y, Yao X: Depletion of intracellular $\mathrm{Ca}^{2+}$ stores sensitizes the flow-induced $\mathrm{Ca}^{2+}$ influx in rat endothelial cells. Circ Res 2003;92:286-292.

27 Qi YX, Qu MJ, Long DK, Liu B, Yao QP, Chien S, Jiang ZL: Rho-GDP dissociation inhibitor alpha downregulated by low shear stress promotes vascular smooth muscle cell migration and apoptosis: a proteomic analysis. Cardiovasc Res 2008;80:114-122.

28 Wang HQ, Bai L, Shen BR, Yan ZQ Jiang ZL: Coculture with endothelial cells enhances vascular smooth muscle cell adhesion and spreading via activation of beta1-integrin and phosphatidylinositol 3-kinase/Akt. Eur J Cell Biol 2007;86:51-62.

29 Wang YH, Yan ZQ, Qi YX, Cheng BB, Wang XD, Zhao D, Shen BR, Jiang ZL: Normal shear stress and vascular smooth muscle cells modulate migration of endothelial cells through histone deacetylase 6 activation and tubulin acetylation. Ann Biomed Eng 2010;38:729-737.

- 30 Chiu JJ, Chen LJ, Lee PL, Lee CI, Lo LW, Usami S, Chien S: Shear stress inhibits adhesion molecule expression in vascular endothelial cells induced by coculture with smooth muscle cells. Blood 2003;101:2667-2674.

- 31 Rezvan A, Ni CW, Alberts-Grill N, Jo H: Animal, in vitro, and ex vivo models of flow-dependent atherosclerosis: role of oxidative stress. Antioxid Redox Sign 2011;15:1433-1448.

32 de Wit C, Roos F, Bolz SS, Kirchhoff S, Kruger O, Willecke K, Pohl U: Impaired conduction of vasodilation along arterioles in connexin40-deficient mice. Circ Res 2000;86:649-655.

-33 DePaola N, Davies PF, Pritchard WF Jr, Florez L, Harbeck N, Polacek DC: Spatial and temporal regulation of gap junction connexin43 in vascular endothelial cells exposed to controlled disturbed flows in vitro. Proc Natl Acad Sci USA 1999;96:3154-3159.

-34 Gabriels JE, Paul DL: Connexin43 is highly localized to sites of disturbed flow in rat aortic endothelium but connexin37 and connexin40 are more uniformly distributed. Circ Res 1998;83:636-643.

- 35 Li G, Rivas P, Bedolla R, Thapa D, Reddick RL, Ghosh R, Kumar AP: Dietary resveratrol prevents development of high-grade prostatic intraepithelial neoplastic lesions: involvement of SIRT1/S6K Axis. Cancer Prev Res 2013;6:27-39.

-36 Cho IR, Koh SS, Malilas W, Srisuttee R, Moon J, Choi YW, Horio Y, Oh S, Chung YH: SIRT1 inhibits proliferation of pancreatic cancer cells expressing pancreatic adenocarcinoma up-regulated factor (PAUF), a novel oncogene, by suppression of $\beta$-catenin. Biochem Biophys Res Commun 2012;423:270-275.

-37 Howitz KT, Bitterman KJ, Cohen HY, Lamming DW, Lavu S, Wood JG, Zipkin RE, Chung P, Kisielewski A, Zhang LL, Scherer B, Sinclair DA: Small molecule activators of sirtuins extend Saccharomyces cerevisiae lifespan. Nature 2003;425:191-196.

-38 Gu J, Wang CQ Fan HH, Ding HY, Xie XL, Xu YM, Wang BY, Huang DJ: Effects of resveratrol on endothelial progenitor cells and their contributions to reendothelialization in intima-injured rats. J Cardiovasc Pharmacol 2006; 47:711-721.

-39 Hsieh TC, Lu XH, Guo JQ, Wu JM: Differential regulation of proliferation, cell cycle control and gene expression in cultured human aortic and pulmonary artery endothelial cells by resveratrol. Inter J Mol Med 2010;26:743-749.

40 Lin MT, Yen ML, Lin CY, Kuo ML: Inhibition of vascular endothelial growth factor-induced angiogenesis by resveratrol through interruption of Src-dependent vascular endothelial cadherin tyrosine phosphorylation. Mol Pharmacol 2003;64:1029-36.

41 Szende B, Tyihák E, Király-Véghely Z: Dose-dependent effect of resveratrol on proliferation and apoptosis in endothelial and tumor cell cultures. Exp Mol Med 2000;32:88-92.

42 Pillarisetti S: A review of Sirt1 and Sirt1 modulators in cardiovascular and metabolic diseases. Recent Pat Cardiovas Drug Discov 2008;3:156-164.

43 Alonso F, Boittin FX, Beny JL, Haefliger JA: Loss of connexin40 is associated with decreased endothelium-dependent relaxations and eNOS levels in the mouse aorta. Am J Physiol Heart Circ Physiol 2010;299:H1365-1373. 